\title{
Data Traffic Modeling in RPAS/UAV Networks with Different Architectures
}

\author{
Andrii Grekhov ${ }^{1}$, Vasyl Kondratiuk ${ }^{2}$ and Svitlana Ilnytska 2,3,*(D) \\ 1 Department of Air Navigation Systems, National Aviation University, 03058 Kyiv, Ukraine; \\ grekhovam@gmail.com \\ 2 Research and Training Center "Aerospace Center", National Aviation University, 03058 Kyiv, Ukraine; \\ kon_vm@ukr.net \\ 3 Institute of Laser and Optoelectronics Intelligent Manufacturing, Wenzhou University, \\ Wenzhou 325035, China \\ * Correspondence: ilnytskasv84@gmail.com; Tel.: +380-979-477-517
}

Citation: Grekhov, A.; Kondratiuk, V.; Ilnytska, S. Data Traffic Modeling in RPAS/UAV Networks with Different Architectures. Modelling 2021, 2, 210-223. https://doi.org/ 10.3390/modelling2020011

Academic Editor: Franco Cicirelli

Received: 8 March 2021

Accepted: 26 March 2021

Published: 28 March 2021

Publisher's Note: MDPI stays neutral with regard to jurisdictional claims in published maps and institutional affiliations.

Copyright: (c) 2021 by the authors. Licensee MDPI, Basel, Switzerland. This article is an open access article distributed under the terms and conditions of the Creative Commons Attribution (CC BY) license (https:// creativecommons.org/licenses/by/ $4.0 /)$.

\begin{abstract}
Deploying of Fifth Generation and Beyond Fifth Generation (5G/B5G) wireless networks will require wider coverage, flexible connectivity, low latency, support for a large number of user devices, and more bandwidth. This article explores the paradigm that Remotely Piloted Air Systems (RPASs) or Unmanned Aerial Vehicles (UAVs) are integrated as a communication platform with cellular networks using radio access. It is important to know the possibilities and ways of such integration for effective interaction with RPASs. This paper studies the issues of ensuring the required Quality of Service (QoS) during heavy traffic and the choice of necessary data transmission modes for this. Models of RPAS communication channels with different architectures were created. The relationships between models' performance and traffic parameters were obtained using the NetCracker Professional 4.1 software. The dependencies of the Average Utilization (AU) on the Transaction Size (TS) were analyzed. The effects of different bandwidths and the Bit Error Rate (BER) were studied. The traffic characteristics in all models were compared.
\end{abstract}

Keywords: remotely piloted air systems (RPAS); unmanned aerial vehicles (UAVs); 5G/B5G; data traffic; transaction size; average utilization; BER; bandwidth

\section{Introduction}

Remotely Piloted Air Systems (RPASs) are playing an increasingly important role in civilian, community, and military purposes. The standards body 3rd Generation Partnership Project (3GPP) is monitoring research on seamless integration of RPASs into Fifth Generation and Beyond Fifth Generation (5G/B5G) cellular networks with an aim to improve them. RPASs can be used as wireless access points or relay nodes to increase cellular terrestrial coverage, as well as for surveillance purposes with greater coverage compared to conventional fixed sensor nodes. The flexible spatial network architecture and mobility of RPASs bring great benefits to 5G/B5G cellular systems when integrated.

At present, it is very important to develop new structures for communication, processing and transmission of information in real time to support communication systems of RPASs/Unmanned Aerial Vehicles (UAVs) with high data transfer rates, helping terrestrial cellular communications in future wireless cellular networks. Therefore, the number of publications devoted to these issues is growing. In recent years, books have been published: On RPAS/UAV networks and communications [1], wireless communications and networking for UAV [2], UAV communications for 5G and beyond [3], and UAV applications over cellular networks for 5G and beyond [4]. Reviews and tutorials are devoted: To wireless communications with UAV [5], UAV cellular communications [6], 5G network availability for operation with drones [7], cellular-connected UAVs [8], UAVs for wireless networks [9], and UAV communications for 5G and beyond [10]. 
In this article, we study a RPAS/UAV-enabled Radio Access Networks (RAN) where the RPAS/UAV is used as an airborne platform supporting communications for terrestrial users, including relaying data from the base station to other RPASs/UAVs and terrestrial users, downlink data transmission to terrestrial users, and collecting uplink data. This multi-mode aerial communication platform is most attractive for a practical RAN with different user requirements for the received data. For the RANs under consideration, four models with different architectures are used, which are of practical interest. In the first model, the base station controls the RPAS within the line of sight and communicates through it with the users of the cellular network. In the second model, the base station communicates with the cellular network via two RPASs to investigate the problem of increasing coverage. The third model is used to study the traffic ramification and operation with a large number of users. The fourth model is the closest to real conditions and contains a complex architecture with the largest number of drones and ground users. The main contributions of this paper are summarized as follows.

First, we offer original models as communication platforms for RPASs/UAVs with cellular networks in various operating conditions, for which the characteristics of data traffic in communication channels have been calculated for the first time.

Secondly, due to the complexity of the research problem for the designed models, we propose an effective approach based on simulation modeling. The term "simulation" means the calculation of the characteristics of a model, changing over time, by reproducing the flow of this process on a computer using the equations of its mathematical model.

Third, for various architectures of communication channels, the results were obtained and compared data transmission quality. From this point of view, the work can be considered as the development of theoretical methods for predicting the behavior of RPAS communication channels in critical conditions.

Fourth, the practical value of the results is that using the obtained dependencies, you can predict the channel's behavior when the transaction size, channel bandwidth and bit error rate change.

The rest of this article is organized as follows. Section 3 presents a research method and algorithms for calculating traffic parameters. Section 4 describes the proposed models and parameters of communication channels. Numerical results are presented in Section 5 . At the end of the article, the results are discussed in Section 6 and conclusions are given in Section 7.

\section{Related Works}

The articles below consider technologies and applications of UAVs in cellular networks, prospective RAN with UAVs, reliable communication for aerial vehicles over cellular networks, and other important issues.

The article [11] analyzes UAV cellular communication and valuates the performance of the downlink command and control channel when supported by either: A traditional cellular network serving one user per transmission time interval, or a multi-user massive Multiple Input Multiple Output (MIMO) network exploiting spatial multiplexing.

A RAN with a UAV acting as an aerial platform for communication with ground users in the modes of data collection, data transmission, or relaying is considered in [12]. Iterative design algorithms of the UAV flight trajectory are proposed there.

A single cell cellular network, several cellular users and several UAVs that upload their collected data to a base station are investigated in [13]. The UAV with a high Signal-toNoise Ratio (SNR) for the User-Infrastructure (U2I) channel downloads the collected data directly to the base station, and the UAV with a low SNR for the U2I channel transmits the data to the nearest UAV through the U2U backbone to ensure required Quality of Service (QoS). The distribution of subchannels and the problem of optimizing the speed of the UAV to maximize the total bandwidth of the uplink are formulated.

The network architecture of Distributed and Multi-layer UAVs (DAMUs) has been presented for 5G/B wireless communication [14]. DAMU architecture due to hierarchical 
flexibility can be reconfigured for a specific deployment and application scenario, taking into account key technical factors. A numerical analysis of the attenuation introduced by typical meteorological conditions is carried out in this paper.

Drone Assisted Vehicular Networks (DAVNs) for the efficient integration of communication and networking technologies for drones and connected vehicles are presented in [15]. A comprehensive DAVN architecture is proposed and it is demonstrated that the performance of automotive networks can be significantly improved with the proposed architecture.

An overview of the Long-Term Evolution (LTE) connectivity for UAVs at low altitude is presented in [16]. Typical requirements and characteristics of airborne communication are determined, the different propagation conditions for UAVs and mobiles on the ground are emphasized, and the results of LTE connection modeling for UAVs are presented.

To ensure collaboration between UAVs and terrestrial users, two solutions have been proposed to mitigate interference in existing LTE networks: Interference suppression and antenna beam selection [17]. It is shown that each of solutions can provide up to $30 \%$ increase in throughput and more than $99 \%$ of radio communication.

A path planning scheme taking into account interference for a network of UAVs connected to cellular communication is proposed in [18]. A deep reinforcement learning algorithm, based on Echo State Network (ESN) cells, is proposed to achieve a trade-off between maximizing energy efficiency, reducing wireless latency and interference in the terrestrial network. Each UAV uses the ESN to determine its optimal path, transmit power, and cell association vector at different locations along its path.

Various communication architectures of the Future Flying Ad-Hoc Network (FANET), including existing wireless technologies, are discussed in [19]. A hybrid wireless communication scheme is proposed to take advantage of the 802.11 high data rate and 802.15.1 low power features. The proposed scheme significantly reduces communication costs and improves network performance in terms of bandwidth and latency. The results of modeling using the Optimized Network Engineering Tool (OPNET) are presented, which additionally confirm the effectiveness of our proposed scheme.

The Internet of Things (IoT) requires high-performance two-way communication between UAVs and their ground pilots/users using a cellular network [20]. An important question is whether an existing cellular network designed for terrestrial users can also effectively cover new UAV users in three-dimensional (3D) space for both uplink and downlink. In practice, there are problems of terrestrial communications interference and a non-uniform three-dimensional antenna gain pattern of a terrestrial base station. The article presents a new 3D model of the system, taking into account UAV users, which offers an analytical basis for determining the characteristics of their 3D uplink/downlink coverage. A 3D analysis of the coverage was carried out taking into account the key parameters of the system, such as the cell load factor, the tilt of the ground base station antenna, the UAV height and the antenna beam width.

Potential 5G beneficiaries and network use are considered in [21]. Three main use cases are considered: Vehicle-to-Vehicle communications, drones, and healthcare. The challenges and disadvantages of current cellular technologies have been investigated and highlighted in relation to these use cases, and ways to overcome these disadvantages in 5G networks have been identified.

UAVs as a component of the IoT can provide sensing and communications in the air due to their advantages in mobility and flexibility. However, the cellular QoS between the UAV and the base station cannot be guaranteed when the UAVs are at the edge of a cell or are experiencing deep fading. The article [22] considers the non-cooperative cellular Internet of the UAV and proposes a joint data transfer protocol, in which the UAV can download sensor data using the UAV relay to provide a better quality of communication service for sensing tasks. The design of the trajectory and control of radio resources that support the joint cellular Internet of the UAV are considered. 
The RPAS/UAV communication requirements must be assessed in the context of an Air Traffic Control (ATC) function [23] (Figure 1). Modeling of channels for UAV communication over cellular networks has been considered in a number of works, since UAV communication is an emerging and underexplored field. Accurate channel parameters are critical to optimizing performance and designing effective UAV communications. Models of path loss and shadowing indicators for a radio channel between UAVs and cellular networks are given in [24]. It is shown that the exponent of path losses decreases as the UAV moves upward, approaching propagation in free space for horizontal distances of up to tens of kilometers at an UAV height of about $100 \mathrm{~m}$.

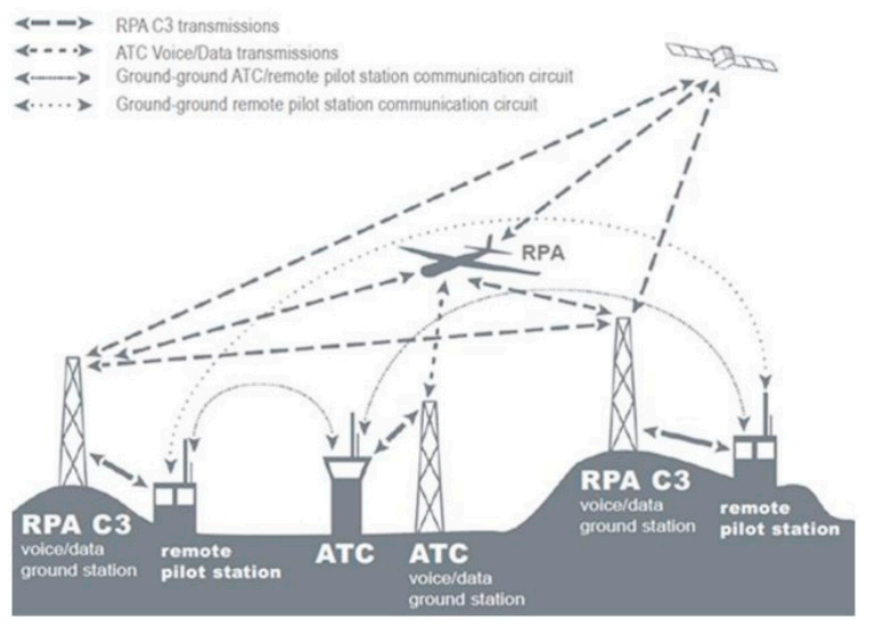

Figure 1. International Civil Aviation Organization (ICAO) aeronautical Remotely Piloted Air System (RPAS) communication links [23].

An overview of the measurement methods proposed for modeling UAV channels using low-altitude platforms, as well as attempts to determine the channel characteristics, is given in [25]. The review also discusses approaches to modeling UAV channels from a modern point of view.

A new Coordinate Multipoint (CoMP) based network architecture for UAV-assisted wireless communications is proposed in [26]. The high mobility of the UAV is used to achieve effective multi-user communication with several UAVs and to reduce interference. The placement and movement of the UAV is optimized using the theory of random matrices.

A new structure for a UAV network with the possibility of mass access, supported by Non-Orthogonal Multiple Access (NOMA), is proposed in [27]. The performance assessment of UAV networks with NOMA is carried out, using stochastic geometry to simulate the position of UAVs and ground users. The joint design of the trajectory and the power distribution for static NOMA users based on a simplified 2D model of a UAV flying at a fixed altitude is investigated. The problem of UAV placement is solved using machine learning methods, when ground users are in roaming, and the UAVs are able to accordingly change their position in three dimensions.

A secured UAV-assisted heterogeneous network environment and seamless Internet protocol prototype is proposed in [28]. UAVs are controlled over the Internet to remove communication barriers over long distances and improve remote sensing. In the new technology, the pilot can control the UAV through secure communication with an end device without any information about network architectures, types of network address translation, and version of the Internet protocol. Network traversal with mobility offers secure communications for UAVs over the public network infrastructure.

An effective technology to improve wireless coverage for remote and disaster-affected areas is UAV radio access, which will be key for providing improved and sustainable coverage in heterogeneous 5G cellular networks. Paper [29] studied edge caching for multiple 
UAV-enabled radio access networks (UAV-RANs) and investigated the improvement in overall spectral efficiency (SE) through efficient edge caching. UAV base stations may not serve directly nearby users, but serve users based on requested content. Based on the SE analysis achieved by the content-oriented UAV-RAN, a hybrid caching strategy is proposed for further improvement the SE.

RPAS communication channels based on IEEE 802.11a, 802.11b, and 802.16 and LTE standards are modeled in our works [30-34].

The articles considered above do not contain quantitative information on the impact of traffic parameters on the operation of RPAS/UAV communication channels. It has not been investigated how quantitatively the channel load can change with an increase in the duration of transactions. How does the bandwidth affect the channel load at the given traffic parameters? How does the increase in bit errors affect? There are no answers to these questions in the literature.

\section{Calculation Method}

The NetCracker Professional 4.1 software [35] was chosen for traffic modeling in proposed models (Figure 2). It uses an analytical simulator based on mathematical equations to predict network behavior. The essence of the applied algorithm is as follows.

Let us denote the internal characteristics of the communication channel, which are calculated at the time $t_{k}$, as $V_{1}\left(t_{k}\right), \ldots, V_{n}\left(t_{k}\right)$; external characteristics at the same moment of time influencing them as $W_{1}\left(t_{k}\right), \ldots, W_{r}\left(t_{k}\right)$, and the average rate of change of internal characteristics in the time interval $\left[t_{k}, t_{k}+1\right]$ as $F_{i}\left(t_{k}, V_{1}\left(t_{k}\right), \ldots, V_{n}\left(t_{k}\right), W_{1}\left(t_{k}\right) \ldots W_{r}\left(t_{k}\right)\right)$, $i=1 \ldots n$. By specifying the values of the internal and external characteristics at the moment $t_{k}$, it is possible to calculate the values of the internal characteristics at the moment $\left(t_{k}+1\right)$. To do this, the segment $\left[t_{0}, T\right]$ is divided into $P$ parts by the points $t_{0}, t_{1}, \ldots$, $t_{k}, \ldots, t_{P}=T$. If the values of internal characteristics at time $t_{0}$ and average rates of the internal characteristics change on each segment $\left[t_{k}, t_{k}+1\right]$ are known, then using the following relations it is possible calculating the values of internal characteristics for all $t_{k}$, $k=1, \ldots, P$ :

$$
V_{i}\left(t_{k}+1\right)=V_{i}\left(t_{k}\right)+\Delta t F_{i}\left(t_{k}, V_{1}\left(t_{k}\right), \ldots, V_{n}\left(t_{k}\right), W_{1}\left(t_{k}\right), \ldots, W_{r}\left(t_{k}\right)\right),
$$

where $i=1, n ; k=0,1, \ldots, P-1$. We modeled such internal characteristics of communication channels as Average Utilization (AU), Travel Time (TT), and the number of dropped packets. Transaction Size (TS), Time Between Transactions (TBT), Bit Error Rate (BER), channel bandwidth, and packet failure probability were chosen as external characteristics that influenced the mentioned internal characteristics. If we consider the functions $Y_{1}(t)$, $\ldots, Y_{n}(t)$ as continuous and having derivatives, then to describe the process, the equations of the following form are used

$$
d V_{i} / d t=F_{i}\left(t, V_{1}, \ldots, V_{n}, W_{1}, \ldots, W_{r}\right),
$$

where $i=1, \ldots, n$. Here, the functions $F_{i}\left(t, V_{1}, \ldots, V_{n}, W_{1}, \ldots, W_{r}\right)$ represent the "instantaneous" rates of change of the corresponding internal characteristics.

To calculate the parameters of the RPAS communication channel, it is necessary to specify the statistical distribution laws for the lengths of transmitted packets $\omega(x)$ and the time intervals (TBT) between them $\omega(t)$. In the proposed models, the Const probability distribution law was used: $\omega(x)=$ Const and $\omega(t)=$ Const. Then the average length of transmitted packets and the average time interval between two neighboring packets are found by the Formulas (3) and (4) correspondently.

$$
T S=\int_{-\infty}^{\infty} x \omega(x) d x .
$$




$$
\text { TBT }=\int_{-\infty}^{\infty} t \omega(t) d t .
$$

The average utilization of the communication channel is given by the formula:

$$
A U=T S / T B T=T S / \mu,
$$

where $\mu=1 / T B T$ is the packet transmission rate over the communication channel. The utilization of the communication channel depends both on the size of the transmitted packets and on the intensity of their generation. If the value of the $\mathrm{AU}$ parameter is greater than the maximum data transmission rate of the communication channel, then some of the transmitted packets will be lost with the probability:

$$
P=1-\frac{A U_{\text {link }}}{A U} .
$$

The average packet travel time through the communication channel is determined by the formula:

$$
T T=\frac{T S}{A U_{\text {link }}} .
$$

\section{Models}

In our previous works [32,33] models of communication channels "Base Station-toRPAS" and "Base Station-to-Satellite-to-RPAS" were built on the basis of 3GPP Wideband Code Division Multiple Access (WCDMA) Standard. Using the MATLAB software, quantitative characteristics of the channels were obtained taking into account the Multipath Rayleigh Fading, the nonlinearity of the satellite transponder, various antenna diameters, and RPAS speeds.

In this work, within the framework of the RPAS-assisted cellular communication paradigm, we first developed models with different architectures for simulating the traffic of Base Station (BS) data exchange with user cellular networks using RPAS.

The BS can be ground or air with two-way traffic both in the control channel and in the payload channel in all models. Flying RPASs have access to cellular infrastructure from the sky. This paradigm allows the establishment of reliable wireless communications with ground cell sites. It is assumed that the BS will not simultaneously work with terrestrial users within the radio Line of Sight (LoS).

Flying RPASs should have antennas with radiation patterns that provide threedimensional coverage for communications with the BS, ground cellular network and other RPASs. Integration of RPASs into a cellular network must provide the necessary QoS, coverage and data transfer rates of the existing cellular network. The proposed model architectures, due to the dynamic mobility and movement of the RPAS, provide many advantages to the existing ground communication system. The flying BS or relay can be provided as required upon request of 5G/B5G cellular systems, which is an attractive operational solution in emergencies, disasters, and search and rescue operations. The RPAS team reliably communicates directly with each other to monitor autonomous flight behavior and prevent collisions in swarms. This architecture is illustrated by the example of Models 2, 3, and 4 (Figure 2). 


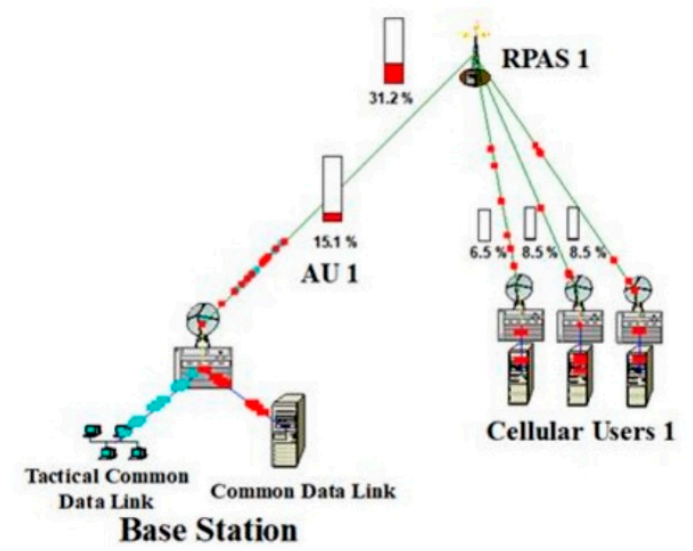

(a)

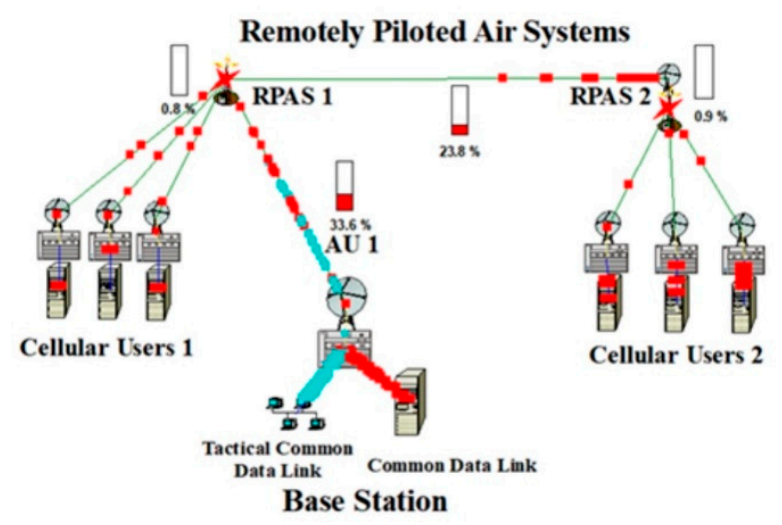

(c)

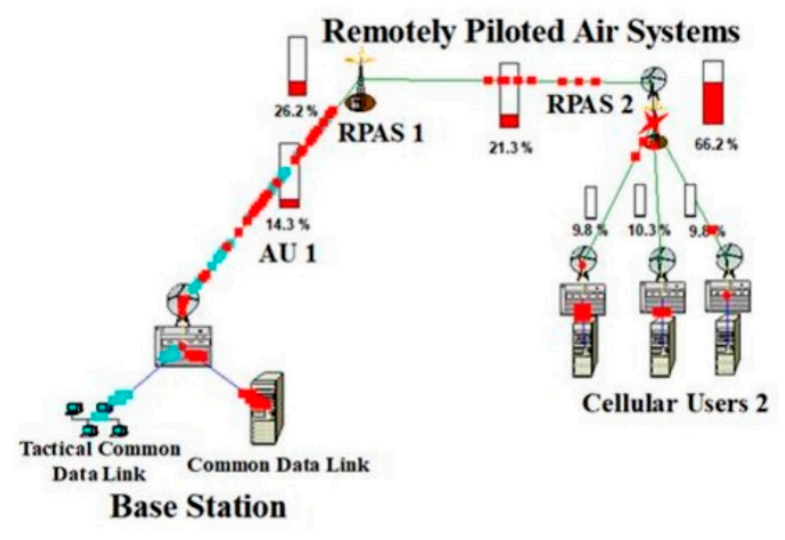

(b)

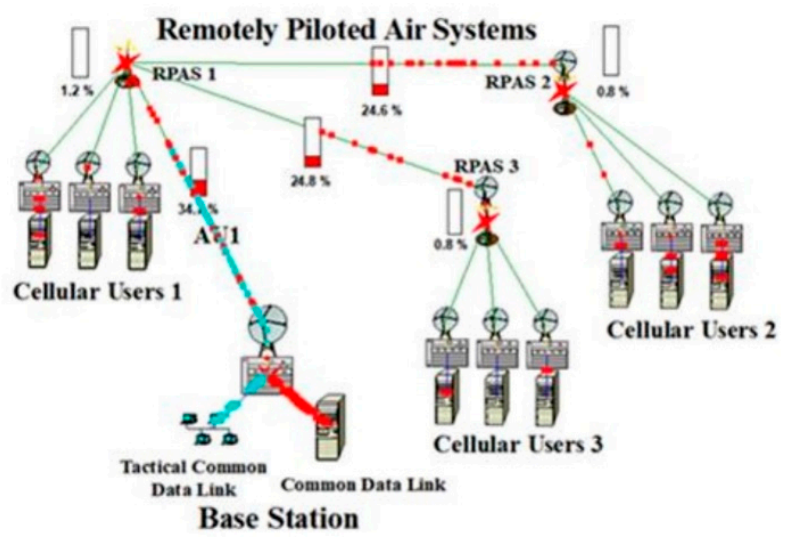

(d)

Figure 2. Architecture of models: (a) Model 1; (b) Model 2; (c) Model 3; and (d) Model 4.

The models' architecture was based on ICAO document [23] and included BS, RPASs, and Cellular Users (CUs). All models (Figure 2) were designed using Professional NetCracker 4.1 software [35] and their parameters are given in Table 1.

In Model 1 "BS-RPAS1-CU3", the BS controls RPAS1 in LoS, and through it communicates with the cellular network, represented by three users. In all models, networks containing 1,3,5, and 7 users were considered, but in this work, for simplicity, data are given for only three users for each RPAS, which does not limit in any way the generality of the consideration.

In Model 2 "BS-RPAS1-RPAS2-CU3", the BS communicates with the cellular network through two RPASs, which increases coverage and reveals the impact of the second RPAS on data traffic.

Model 3 “BS-RPAS1-CU3-RPAS2-CU3” adds users to RPAS1 for simulation of traffic ramification to work with more users and increase the BS coverage.

Model 4 "BS-RPAS1-CU3-RPAS2-CU3-RPAS3-CU3" considers an even more complex architecture with an addition of RPAS3 and CU 3, which further increases the BS coverage and increases the load on RPAS1 (Figure 3). All wireless connections of the BS, RPASs and CUs are selected to be $10 \mathrm{~km}$. 
Table 1. Parameters of Models.

\begin{tabular}{|c|c|c|c|}
\hline Parameters $\rightarrow$ & \multirow{2}{*}{ Bandwidth (Mbps) } & \multirow{2}{*}{ Length (m) } & \multirow{2}{*}{ BER $(\%)$} \\
\hline Model Elements $\downarrow$ & & & \\
\hline \multicolumn{4}{|c|}{ Model 1: “BS-RPAS1-CU3” } \\
\hline Base Station & & & \\
\hline Tactical Data (TD) Workgroup & 10 & - & - \\
\hline Common Data (CD) Server & 10 & - & - \\
\hline TD—Switch link & 10 & 1 & 0 \\
\hline CD—Switch link & 10 & 1 & 0 \\
\hline Switch & 1000 & - & - \\
\hline Switch-Antenna link & 44.736 & 10 & 0 \\
\hline Antenna & 1000 & - & - \\
\hline BS_RPAS 1 wireless link & $2.048-44.736$ & $10^{5}$ & $0-0.05$ \\
\hline RPAS 1 & 1000 & - & - \\
\hline $\begin{array}{c}\text { RPAS } 1 \text { - CUs } 1 \text { wireless links } \\
\text { CUs } 1\end{array}$ & 44.736 & $10^{5}$ & 0 \\
\hline Antenna & 1000 & - & - \\
\hline Antenna-Switch link & 44.736 & 10 & 0 \\
\hline Switch & 1000 & - & - \\
\hline Switch-Server link & 10 & 1 & 0 \\
\hline Server & 10 & - & - \\
\hline \multicolumn{4}{|c|}{ Model 2: “BS-RPAS1-RPAS2-CU3” (in addition to Model 1) } \\
\hline RPAS 1-RPAS 2 wireless link & 44.736 & $10^{5}$ & 0 \\
\hline Antenna & 1000 & - & - \\
\hline RPAS 2 & 1000 & - & - \\
\hline \multicolumn{4}{|c|}{ Model 3: “BS-RPAS1-CU3-RPAS2-CU3” (in addition to Model 2) } \\
\hline $\begin{array}{c}\text { RPAS1-CUs } 1 \text { wireless links } \\
\text { CUs } 1\end{array}$ & 44.736 & $10^{5}$ & 0 \\
\hline \multicolumn{4}{|c|}{ Model 4: “BS-RPAS1-CU3-RPAS2-CU3-RPAS3-CU3” (in addition to Model 3) } \\
\hline RPAS 1-RPAS 3 wireless link & 44.736 & $10^{5}$ & 0 \\
\hline $\begin{array}{c}\text { RPAS 3-CUs } 3 \text { wireless links } \\
\text { CUs } 3\end{array}$ & 44.736 & $10^{5}$ & 0 \\
\hline
\end{tabular}

The wireless architecture of connecting the BS to the RPAS1 and each of the RPAS to the $\mathrm{CU}$ results in more reliable LoS channels. In addition, our models incorporate RPASs space diversity to reduce mutual interferences. In reality, there are effects such as fading, shading and loss along the way that should be considered. Only the free-space channel model lacks fading and shading effects and is suitable for rural areas where the LoS assumption is valid for high-altitude RPAS and ground stations. However, in urban environments, low-altitude RPAS can detect channels other than LoS. Therefore, it is necessary to look for ways to properly match the propagation conditions taking into account the propagation loss in space.

Mentioned effects can lead to a decrease in the data transmission rate and an increase in the number of bit errors in messages. For a quantitative assessment of the consequences of this, we simulated data transmission with different rates (Figure 4) and the number of bit errors (Figure 5) for all models.

For RPAS data transmission, it is necessary to organize Command, Control, and Communication (C3) data traffic (Figure 1) in accordance with the ICAO circular [23]. The C3 data traffic consists of a Tactical Data channel (Command and Control) for RPAS flight control and a Common Data channel for transmitting payload data. Command and Control communication without payload data refers to time-critical control and safety commands for maintaining flights and includes navigation, waypoint updates, telemetry reports, and Air Traffic Control communications. The reliability requirement for this is less than $10^{-6}$ bit error rate. Payload transmission refers to all information of the RPAS/UAV mission and 
can include the transmission of data from users of the cellular network, remote multisensor data (radar data, optical, infrared systems, etc.), and transmit real-time video at a high data rate in the surveillance operation.

Traffic with File Transfer Protocol (FTP) profile for Tactical Data (TS $=100$ Kbits and TBT = $1 \mathrm{~s}$ with Const distribution laws) and Local Area Network (inter LAN) profile for Common Data (TS and TBT with Const distribution laws, TBT = $1 \mathrm{~s}$ ) has been defined for the designed models with topology according to Figure 2. Command, Control, and Communication traffic is carried out as two-way communication. RPAS/UAV communication requirements were selected in accordance with the cellular communications [8] and ICAO requirements [36].

\section{Results}

This section presents the calculated dependencies of the average utilization for the "BS-RPAS1" link (AU1) on the TS parameter, the link bandwidth and the BER. The average channel utilization refers to the average traffic on a given channel as a percentage of a total channel bandwidth. The channel bandwidth is the amount of information in bits per second that a channel can pass over a certain time period. In addition to the average utilization, such parameters as message travel time, the number of dropped packets, and the average workload were also calculated. For simplicity, this article provides data for the average utilization only.

It is necessary to find how the architecture of the models is related to the quantitative characteristics of traffic. How can adding of one or two new RPASs change traffic? How does traffic change across models with different architectures in response to increasing transaction sizes? At what size of transactions is the channel closed? Does this happen for the same values in different architectures?

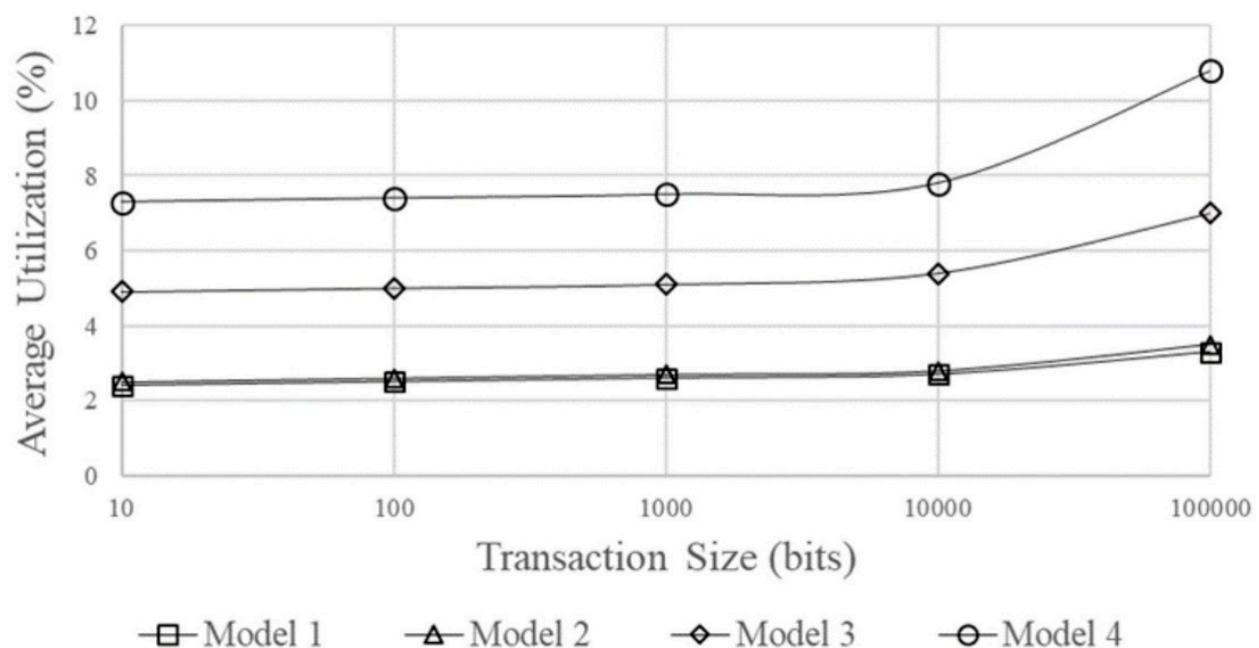

Figure 3. Dependencies of Average Utilization (AU) for AU1 link on Common Data Transaction Size (TS) (Tactical Data-File Transfer Protocol (FTP), TS = 100 Kbits, Time Between Transactions $(\mathrm{TBT})=1 \mathrm{~s}$, Const law; Common Data-Local Area Network (inter LAN), TBT = $1 \mathrm{~s}$, Const law; and Bit Error Rate $(\mathrm{BER})=0 \%)$.

All distances were considered to be equal to $10 \mathrm{~km}$, as indicated in Table 1 . The goal was to find out the average AU1 link utilization for different models. Figure 3 shows the dependencies of the AU parameters for the "BS-RPAS1" channels on the size of the Common Data transactions for different models. At the same time, Tactical Data traffic remained constant with TS $=100$ Kbits. Messages of both types of traffic are sent every second. The nature of the dependencies is approximately the same for all models. The AU values practically do not change for TS parameter values from 10 bits to $10 \mathrm{Kbit}$, increasing only for TS $=100$ Kbits. The increase in the AU parameter occurs as the transition from 
Model 1 to Model 4, that is, as the architecture of the models becomes more complex. When TS > 100 Kbits, the channel is closed for all models. The transmission of Common Data becomes impossible in the case of the selected transmission conditions for Tactical Data traffic. The results for Models 1 and 2 are practically the same (for example, $\approx 2.5 \%$ and $\approx 2.7 \%$, respectively, for TC $=1000$ bits), but with increasing complexity of the model architecture, the AU parameter becomes equal to $\approx 5.7 \%$ (Model 3 ) and $\approx 7.5 \%$ (Model 4 ). The increase in the load of the AU1 channel during the transition from Model 1 to Model 4 occurs almost three times.

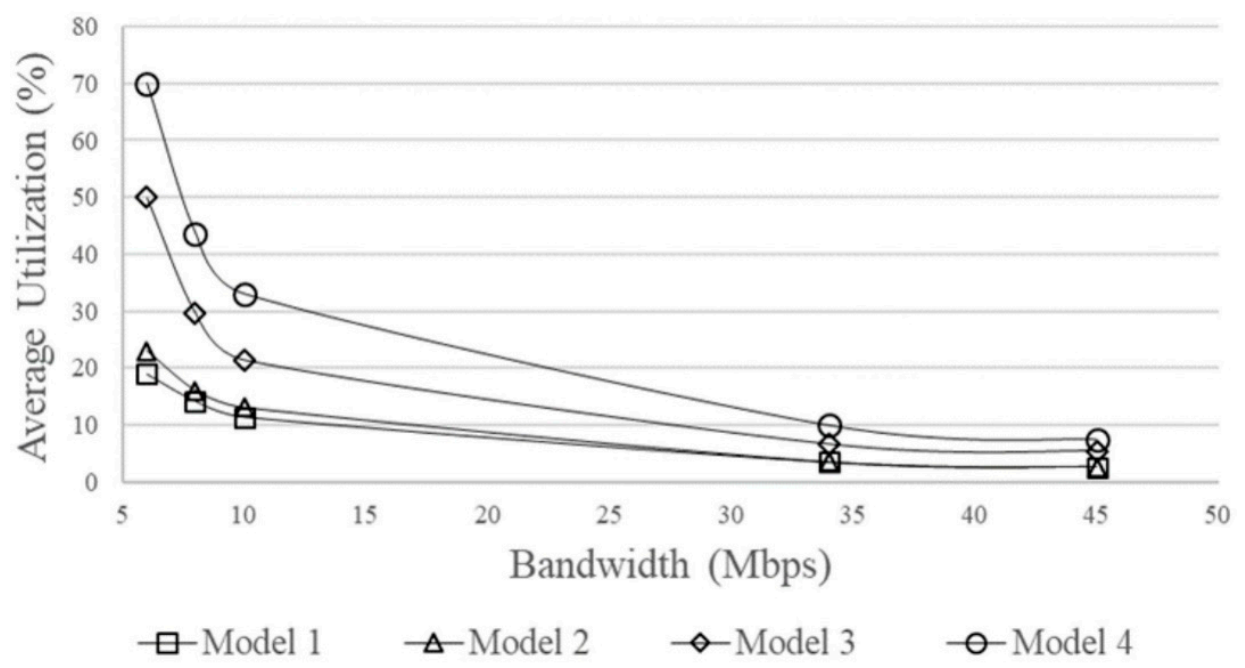

Figure 4. Dependencies of AU on AU1 link bandwidth (Tactical Data-FTP, TS $=1 \mathrm{Kbits}, \mathrm{TBT}=1 \mathrm{~s}$, Const law; Common Data-inter LAN, TS = 10 Kbits, TBT = $1 \mathrm{~s}$, Const law; and BER =0\%).

The established requirements for the cellular communication of the RPASs/UAVs [8,36] correspond to the data transfer rate over the Command and Control channel $\sim 100 \mathrm{Kbps}$ and the payload channel $\sim 50 \mathrm{Mbps}$. That is why when obtaining the dependencies shown in Figure 3, for Tactical Data the parameters TS $=100 \mathrm{Kbits}, \mathrm{TBT}=1 \mathrm{~s}$ were chosen. At the same time, the data transfer rate or bandwidth for Tactical and Common Data in all network branches was T3 (44.736 Mbps), as indicated in Table 1. However, in reality, the data transfer rate may decrease due to different reasons. As the bandwidth decreases, less and less information can pass through the channel per unit of time. How can a change in data transfer rate affect network load in models with different architectures? With what data transfer rates models can operate? The answers to these questions are contained in Figure 4 .

The dependencies of the AU parameter on AU1 link bandwidth for all models (Figure 2) are shown in Figure 4. The bandwidth ranged from $6 \mathrm{Mbps}$ to E3 (34.368 Mbps) and T3 (44.736 Mbps). Dependencies are given for TS $=1 \mathrm{Kbits}$ for Tactical Data traffic and TS $=10 \mathrm{Kbits}$ for Common Data traffic. This is due to the fact that it is necessary to simultaneously observe the data transmission in all models, which is difficult to ensure at large values of the TS parameters for both channels. The AU parameter increases with decreasing the bandwidth and at $6 \mathrm{Mbps}$ bandwidth reaches $\approx 70 \%$ for Model $4, \approx 50 \%$ for Model 3, $\approx 23 \%$ for Model 2 , and $\approx 19 \%$ for Model 1 . For T3 and E3 bandwidths AU values for all models do not exceed $\approx 10 \%$. However, as the bandwidth decreases, a significant increase in the AU parameter is observed, which makes it practically impossible to transfer data for Models 3 and 5 at the bandwidth below $\approx 10 \mathrm{Mbps}$. The results for Models 1 and 2 are very close and show the data transfer capability of such models over a wide range of data rates. 


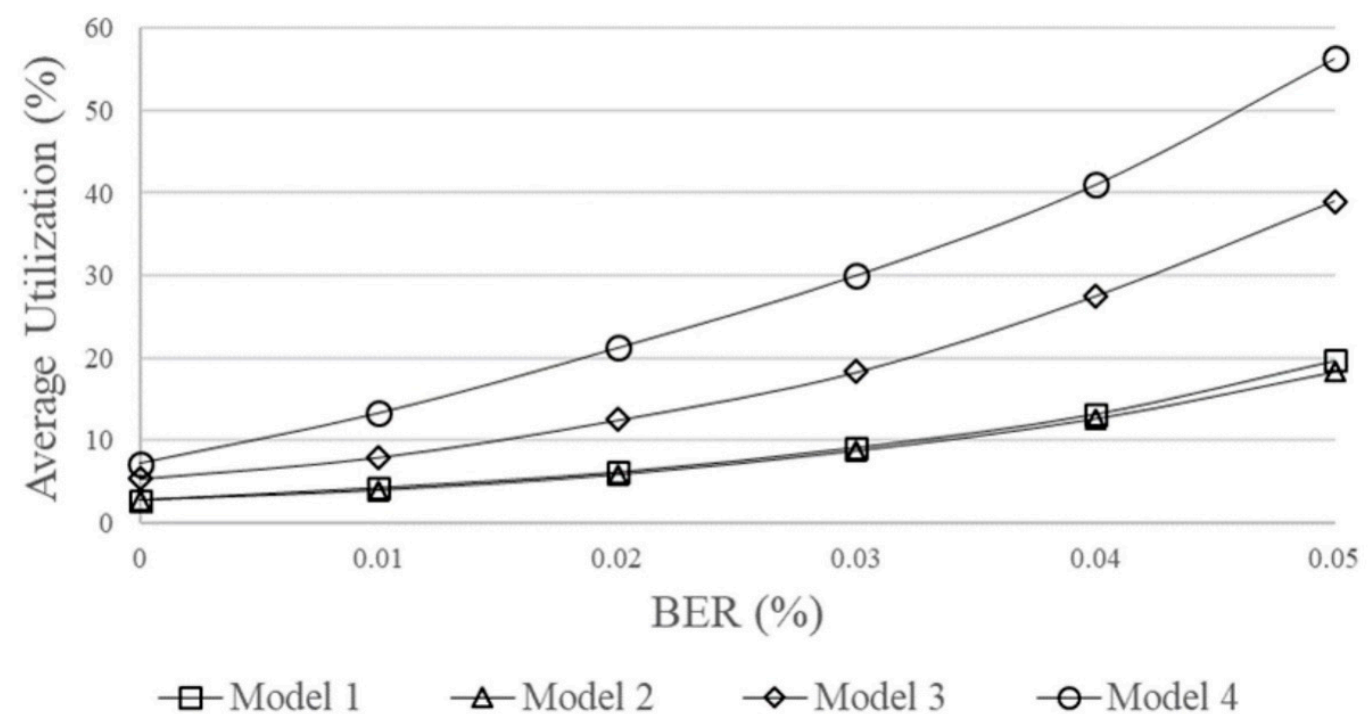

Figure 5. Dependencies of AU on AU1 link BER (Tactical Data-FTP, TS $=1$ Kbits, TBT = $1 \mathrm{~s}$, Const law; Common Data-inter LAN, TS = 10 Kbits, TBT = $1 \mathrm{~s}$, Const law; and BER = 0\%).

The BER measured after Forward Error Correction (FEC) should be less than $10^{-6}$ in accordance with ICAO requirements [36,37]. Figure 5 shows the dependencies of the AU parameter on the BER for the "BS-RPAS1" channels of all models. The dependencies are given for TS $=1$ Kbits for Tactical Data traffic and TS $=10$ Kbits for Common Data traffic as in the case of Figure 4 . The BER value for channels without additional error correction is usually $10^{-4}-10^{-6}$. Bit distortion occurs both due to the presence of noise in the channel and due to the distortion of the waveform in channels with limited bandwidth. The BER value of $0.01 \%$ in Figure 5 corresponds to a value of $10^{-4}$. The data presented in Figure 5 indicate a high sensitivity of channels to bit errors. As in previous cases, the results for Models 1 and 2 are very close. The results indicate that it is necessary to use effective error correction techniques in the case of complex networks (Models 3 and 4).

\section{Discussion}

The RPAS in practical use often requires communication that goes beyond visual line of sight. Mobile networks offer the opportunity to use the RPAS outside of visual LoS with wide area, high speed, and secure wireless connections.

This work is devoted to the development of theoretical methods for predicting the behavior of RPAS communication channels when integrating with cellular networks. In our previous works ([30-34] and references therein), we studied the transmission of messages using RPASs/UAVs in direct and indirect visibility, through terrestrial networks without and with the use of satellites. The research was based on the simulation of communication channels using Matlab and NetCracker software. IEEE 802.11a, 802.11b, and 802.16 [30,31] and LTE Standards $[32,33]$ have been used in the various models. The main purpose of these studies was to obtain quantitative characteristics of RPAS communication channels and to develop methods for calculating the parameters of communication channels.

The articles considered in the introduction demonstrate the achievements in the field of RPASs/UAVs communications. However, we could not find quantitative information on the influence of traffic parameters on the exchange of data between the base station and users of cellular networks through the RPAS/UAV. How does the transactions size affect channel loading? How does the bandwidth affect the channel utilization for the given traffic parameters? What and how affects the quality of service, namely the increase in the number of bit errors? There are no answers to such questions in the literature, so this article considers three main aspects: The different architecture of channel models, an 
algorithm for calculating the parameters of communication channels, and the application of the results to assess the quality of data transmission.

From data of Figure 3 (dependencies on the transaction size) it follows that in a wide range of the TS parameter (from 10 bits to $10 \mathrm{Kbits}$ ), the AU values practically do not change for all models. At the same time, the complexity of the network architecture leads to an increase in the network load by more than three times.

Figure 4 (dependencies on the bandwidth) shows that for reliable data transmission you should use a bandwidth not lower than T3 $(44.736 \mathrm{Mbps})$ for all models. In this case the network load will not exceed $\approx 10 \%$. In complex networks, efficient data transmission is practically impossible at bandwidths below $10 \mathrm{Mbps}$ due to a significant increase in network utilization.

At BER $=0 \%$ (Figure 5), the AU values for Models 1 and 2 coincide $(\approx 2.7 \%)$, for Model 3 is of the order of $\approx 5.4 \%$, and for Model 4 it reaches $\approx 7.2 \%$. As the BER increases, the differences between the models increase, and the situation is most unfavorable for Model 4. Figure 5 is relevant for understanding the conditions for achieving the required level of QoS in the network.

In addition to the above models, we considered Model 1 (Figure 2), containing $\mathrm{N}$ users of the cellular network. It was important to analyze this in order to understand what to expect from a significant increase in users' number and how this increases the load in the RPAS communication channel. Model 1 was considered for the cases of $N=1,3,5,7$, and 10 users, for which values similar to those shown in the Figures $3-5$ have been calculated. At the same time, all links bandwidth, in contrast to the data of Table 1, were taken to be equal to $10 \mathrm{Mbps}$ (which is almost 5 times less), and the FTP traffic in the Control and Command channel was $10 \mathrm{Kbits}$ (which is 10 times more than discussed above). This choice of parameters was interesting for analyzing the behavior of "insufficiently fast" networks with significant traffic volumes. For brevity, these data are not presented in full here. Let us just say that, for example, doubling the number of users from $\mathrm{N}=5$ to $\mathrm{N}=10$ leads to a twofold increase in the parameter AU for the link "BS-RPAS1" (TS = 1000 bits and other parameters similar to those discussed above).

In general, our research shows that using the obtained data on traffic characteristics (Figures 3-5), it is possible to choose the right operating conditions and successfully exchange data between the base station and users of remote cellular networks using RPAS/UAV integration.

In the future, we will expand our research to RPAS satellite radio access networks, coordinated data transfers between ground base stations and predicting channel behavior in critical conditions. In our next work, we will present the results obtained for models containing instead of RPAS1 (Figure 2) the low-orbit satellite Iridium.

NetCracker software tools allow you to create a network, carry out the necessary simulation experiments, determine the final characteristics, change the topology and modify the network equipment for further improvement and development. The capabilities of the NetCracker software for predicting traffic parameters were tested in our work [38]. The obtained dependencies of the message transit time (1.4-1.9 s) on the number of satellites and aircraft were experimentally confirmed by Aireon [39], which provided air traffic surveillance data to its partners. Tracking more than 10,000 aircraft with ADS-B 1090 Extended Squitter receivers for Iridium NEXT satellites, the system delivered data to air traffic control centers with a delay of less than $1.5 \mathrm{~s}$ [40].

\section{Conclusions}

The presented research is devoted to obtaining quantitative characteristics of RPAS/ UAV traffic parameters for radio access technology to cellular networks. Models of RPAS/UAV integration are based on the ICAO recommendations. Base station data transmission channels have different architectures, for which the RPAS/UAV traffic parameters were first obtained. The dependencies of the average channel utilization on the transaction size, the effect of different bandwidth, and the effect of the bit error rate were obtained 
and analyzed. A quantitative comparison of traffic characteristics in models with different topologies was carried out.

It was discovered that the AU values practically do not change for the considered models when varying the TS parameter (from 10 bits to $10 \mathrm{Kbits}$ ), which means messages of bigger size can be transmitted with the same efficiency. However, for reliable data transmission the one should use a bandwidth not lower than T3 (44.736 Mbps) for all models (to avoid network load higher than $10 \%$ ). At zero BER all considered models has $\mathrm{AU}$ values less than $8 \%$. However, with the BER increase, the situation becomes the most unfavorable for Model 4 . The obtained data allow us to judge about the provision of the required quality of service with heavy traffic and about the choice of the necessary data transmission modes for this. With appropriate communication strategies, an RPAS/UAV platform can provide a viable relay or broadcast node for high-bandwidth communications.

Author Contributions: Conceptualization, A.G., V.K. and S.I.; methodology, S.I. and A.G.; validation, S.I., A.G. and V.K.; investigation, A.G. and S.I.; resources, V.K.; writing-original draft preparation, A.G.; writing-review and editing, S.I.; supervision, project administration and funding acquisition, V.K. All authors have read and agreed to the published version of the manuscript.

Funding: This research received no external funding.

Data Availability Statement: Not applicable.

Conflicts of Interest: The authors declare no conflict of interest.

\section{References}

1. Namuduri, K.; Chaumette, S.; Kim, J.H.; Sterbenz, J.P.G. (Eds.) UAV Networks and Communications; Cambridge University Press: Cambridge, UK, 2017. [CrossRef]

2. Saad, W.; Bennis, M.; Mozaffari, M.; Lin, X. Wireless Communications and Networking for Unmanned Aerial Vehicles; Cambridge University Press: Cambridge, UK, 2020. [CrossRef]

3. Yong, Z.; Guvenc, I.; Zhang, R.; Gerazi, G.; Matolak, D.W. UAV Communications for 5G and Beyond; Wiley-IEEE Press: Hoboken, NJ, USA, 2020. [CrossRef]

4. Zhang, H.; Song, L.; Han, Z. Unmanned Aerial Vehicle Applications over Cellular Networks for 5G and Beyond; Springer International Publishing: Cham, Switzerland, 2020. [CrossRef]

5. Zeng, Y.; Zhang, R.; Lim, T.J. Wireless Communications with Unmanned Aerial Vehicles: Opportunities and Challenges. IEEE Commun. Mag. 2016, 54. [CrossRef]

6. Fotouhi, A.; Qiang, H.; Ding, M.; Hassan, M.; Giordano, L.G.; Garcia-Rodriguez, A.; Yuan, J. Survey on UAV Cellular Communications: Practical Aspects, Standardization Advancements, Regulation, and Security Challenges. IEEE Commun. Surv. Tutor. 2019, 21, 3417-3442. [CrossRef]

7. Bor-Yaliniz, I.; Salem, M.; Senerath, G.; Yanikomeroglu, H. Is 5G Ready for Drones: A Look into Contemporary and Prospective Wireless Networks from a Standardization Perspective. IEEE Wirel. Commun. 2019, 26, 19-27. [CrossRef]

8. Mishra, D.; Natalizio, E. A Survey on Cellular-Connected UAVs: Design Challenges, Enabling 5G/B5G Innovations, and Experimental Advancements. arXiv 2020, arXiv:abs/2005.00781.

9. Mozaffari, M.; Saad, W.; Bennis, M.; Nam, Y.-H.; Debbah, M. A Tutorial on UAVs for Wireless Networks: Applications, Challenges, and Open Problems. IEEE Commun. Surv. Tutor. 2019, 21, 2334-2360. [CrossRef]

10. Zeng, Y.; Wu, Q.; Zhang, R. Accessing from the Sky: A Tutorial on UAV Communications for 5G and Beyond. Proc. IEEE 2019, 107, 2327-2375. [CrossRef]

11. Geraci, G.; Garcia-Rodriguez, A.; Giordano, L.G.; Lopez-Perez, D.; Bjornson, E. Understanding UAV Cellular Communications: From Existing Networks to Massive MIMO. IEEE Access 2018, 6, 67853-67865. [CrossRef]

12. Zhang, J.; Zeng, Y.; Zhang, R. UAV-Enabled Radio Access Network: Multi-Mode Communication and Trajectory Design. IEEE Trans. on Signal Process. 2018, 66, 5269-5284. [CrossRef]

13. Zhang, S.; Zhang, H.; Di, B.; Song, L. Cellular UAV-to-X Communications: Design and Optimization for Multi-UAV Networks. arXiv 2018, arXiv:abs /1801.05000. [CrossRef]

14. Huo, Y.; Dong, X.; Lu, T.; Xu, W.; Yuen, M. Distributed and Multi-Layer UAV Network for the Next-Generation Wireless Communication. arXiv 2018, arXiv:abs/1805.01534.

15. Shi, W.; Zhou, H.; Li, J.; Xu, W.; Zhang, N.; Shen, X. Drone Assisted Vehicular Networks: Architecture, Challenges and Opportunities. IEEE Network 2018, 32, 130-137. [CrossRef]

16. Lin, X.; Yajnanarayana, V.; Muruganathan, S.D.; Gao, S.; Asplund, H.; Maattanen, H.-L.; Bergstrom, M.; Euler, S.; Wang, Y.-P.E. The Sky Is Not the Limit: LTE for Unmanned Aerial Vehicles. IEEE Commun. Mag. 2018, 56, 204-210. [CrossRef] 
17. Nguyen, H.C.; Amorim, R.; Wigard, J.; Kovacs, I.Z.; Sorensen, T.B.; Mogensen, P.E. How to Ensure Reliable Connectivity for Aerial Vehicles over Cellular Networks. IEEE Access 2018, 6, 12304-12317. [CrossRef]

18. Challita, U.; Saad, W.; Bettstetter, C. Interference Management for Cellular-Connected UAVs: A Deep Reinforcement Learning Approach. IEEE Trans. Wirel. Commun. 2019, 18, 2125-2140. [CrossRef]

19. Khan, M.A.; Qureshi, I.M.; Khanzada, F. A Hybrid Communication Scheme for Efficient and Low-Cost Deployment of Future Flying Ad-Hoc Network (FANET). Drones 2019, 3, 16. [CrossRef]

20. Lyu, J.; Zhang, R. Network-Connected UAV: 3-D System Modeling and Coverage Performance Analysis. IEEE Internet Things J. 2019, 6, 7048-7060. [CrossRef]

21. Ullah, H.; Nair, N.G.; Moore, A.; Nugent, C.; Muschamp, P.; Cuevas, M. 5G Communication: An Overview of Vehicle-toEverything, Drones, and Healthcare Use-Cases. IEEE Access 2019, 7, 37251-37268. [CrossRef]

22. Zhang, H.; Song, L.; Han, Z.; Poor, H.V. Cooperation Techniques for A Cellular Internet of Unmanned Aerial Vehicles. arXiv 2019, arXiv:abs/1904.01257. [CrossRef]

23. ICAO. Circular 328-AN/190; Unmanned Aircraft Systems (UAS): Montréal, QC, Canada, 2011.

24. De Amorim, R.M.; Nguyen, H.C.; Mogensen, P.E.; Kovacs, I.Z.; Wigard, J.; Sørensen, T.B. Radio Channel Modeling for UAV Communication over Cellular Networks. IEEE Wirel. Commun. Lett. 2017, 6, 514-517. [CrossRef]

25. Khuwaja, A.A.; Chen, Y.; Zhao, N.; Alouini, M.-S.; Dobbins, P. A Survey of Channel Modeling for Uav Communications. IEEE Commun. Surv. Tutor. 2018, 20, 2804-2821. [CrossRef]

26. Liu, L.; Zhang, S.; Zhang, R. CoMP in the Sky: UAV Placement and Movement Optimization for Multi-User Communications. IEEE Trans. Commun. 2019, 67, 5645-5658. [CrossRef]

27. Liu, Y.; Qin, Z.; Cai, Y.; Gao, Y.; Li, G.Y.; Nallanathan, A. UAV Communications Based on Non-Orthogonal Multiple Access. arXiv 2018, arXiv:abs /1809.05767. [CrossRef]

28. Aljehani, M.; Inoue, M.; Watanbe, A.; Yokemura, T.; Ogyu, F.; Iida, H. UAV Communication System Integrated into Network Traversal with Mobility. SN Appl. Sci. 2020, 2, 1057. [CrossRef]

29. Zhou, F.; Wang, N.; Luo, G.; Fan, L.; Chen, W. Edge Caching in Multi-UAV-Enabled Radio Access Networks: 3D Modeling and Spectral Efficiency Optimization. IEEE Trans. Signal Inf. Process. Over Netw. 2020, 6, 329-341. [CrossRef]

30. Grekhov, A.M. Recent Advances in Satellite Aeronautical Communications Modeling. In Advances in Wireless Technologies and Telecommunication; IGI Global: Hershey, PA, USA, 2019. [CrossRef]

31. Grekhov, A.; Kondratiuk, V.; Ilnitska, S. RPAS Satellite Communication Channel Based on IEEE 802.11b Standard. Transp. Aerosp. Eng. 2019, 7, 32-40. [CrossRef]

32. Grekhov, A.; Kondratiuk, V.; Ilnitska, S. RPAS Satellite Communication Channel Based on Long-Term Evolution (LTE) Standard. Transp. Aerosp. Eng. 2020, 8, 1-14. [CrossRef]

33. Grekhov, A.; Kondratiuk, V.; Ilnytska, S. RPAS Communication Channels Based on WCDMA 3GPP Standard. Aviation 2020, 24, 42-49. [CrossRef]

34. Ilnytska, S.I.; Li, F.; Grekhov, A.; Kondratiuk, V. Loss Estimation for Network-Connected UAV/RPAS Communications. IEEE Access 2020, 8, 137702-137710. [CrossRef]

35. Netcracer. Available online: https:/ / www.netcracker.com/ (accessed on 26 February 2021).

36. 3GPP TR 36.777. Technical Specification Group Radio Access Network: Study on Enhanced LTE Support for Aerial Vehicles; V15.0.0; December 2017. Available online: https:/ / www.3gpp.org/ftp/Specs/archive/36_series/36.777/ (accessed on 28 March 2021).

37. ICAO. BER Estimation through Packet Error Rate Measurement. ACP-WG-S_Web Meeting 5 WP04_Error measurement.cdoc. Available online: https://www.icao.int/safety/acp/ACPWGF/ACP-WG-S-Web\%20Meeting\%205/ACP-WG-S_WP04ErrorMeasurment_r11\%20\%20(2).docx (accessed on 26 February 2021).

38. Kharchenko, V.; Bo, W.; Grekhov, A.; Kovalenko, M. Investigation of ADS-B Messages Traffic Via Satellite Communication Channel. Proc. Natl. Aviat. Univ. 2014, 61, 7-13. [CrossRef]

39. Collins, M. First Space-Based ADS-B Satellites in Orbit. AOPA, 18 January 2017. Available online: https:/ /www.aopa.org/newsand-media/all-news/2017/january/18/first-space-based-ads-b-satellites-in-orbit (accessed on 28 March 2021).

40. Iridium-NEXT. Spaceflight101. Available online: https://spaceflight101.com/spacecraft/iridium-next/ (accessed on 26 February 2021). 